\title{
ESTUDIO CUALITATIVO DE LOS PROCESOS DE GRAMATICALIZACIÓN, CAMBIO CONSTRUCCIONAL Y CONSTRUCCIONALIZACIÓN EN LAS CONSTRUCCIONES PROGRESIVAS DEL INGLÉS ${ }^{1}$
}

\author{
Carlos van Arkel-Simón \\ Universidad Nacional de Educación a Distancia \\ cvanarkel@la-laguna.uned.es
}

\begin{abstract}
RESUMEN: En este artículo se analizan cualitativamente determinados procesos de cambio gramatical que experimentan las construcciones progresivas del inglés a lo largo de su desarrollo diacrónico. Más concretamente, se estudian los procesos de gramaticalización, tanto de carácter primario como secundario, en que devienen las formas progresivas, así como los fenómenos de cambio construccional y construccionalización que parecen tener lugar en la evolución de estas estructuras. Se lleva a cabo un análisis cualitativo estandarizado, a partir de una muestra del conjunto de corpus York-Toronto-Helsinki-Penn, mediante el cual se prueba la existencia de una relación entre los procesos de gramaticalización primaria y los cambios construccionales, así como entre los fenómenos de gramaticalización secundaria y la construccionalización. Finalmente, el concepto de gradiente lingüistico, aplicado a estos procesos de cambio gramatical, explica tentativamente la forma en la que estos fenómenos de cambio podrían encontrarse latentes en las formas progresivas en todos los periodos de la lengua.
\end{abstract}

PALABRAS CLAVE: lingüistica histórica inglesa, gramaticalización, cambio construccional, construccionalización, lingüistica del corpus, metodología cualitativa.

1. El contenido de este artículo ha sido elaborado a partir del manuscrito de mi tesis doctoral titulada El cambio gramatical en las construcciones progresivas del inglés. 


\title{
QUALITATIVE STUDY OF GRAMMATICALIZATION, CONSTRUCTIONAL CHANGE AND CONSTRUCTIONALIZATION PHENOMENA IN THE ENGLISH PROGRESSIVE CONSTRUCTION
}

\begin{abstract}
In this paper we examine qualitatively certain grammatical change phenomena undergone by English progressive constructions during their diachronic development. More specifically, we study attested primary and secondary grammaticalization processes, and estimated constructional changes and constructionalization phenomena in the evolution of these structures. We implement a qualitative standardized analysis, based on a sample drawn from the family of corpora York-Toronto-Helsinki-Penn, that demonstrates a relationship between primary grammaticalization and constructional changes, and between secondary grammaticalization and constructionalization. Finally, the concept of linguistic gradient, applied to these grammatical change phenomena, tentatively explains how these processes apparently lie in a latent state in the progressive construction in all periods of the English language.

KEYWORDS: English Historical Linguistics, Grammaticalization, Constructional Change, Constructionalization, Corpus Linguistics, Qualitative Methods.
\end{abstract}

\section{ETUDE QUALITATIVE DES PROCESSUS DE GRAMMATICALISATION, DE CHANGEMENT CONSTRUCTION ET DE CONSTRUCTIONALISATION DANS LES CONSTRUCTIONS PROGRESSIVES DE L'ANGLAIS}

\footnotetext{
RÉSUMÉ : Cet article analyse qualitativement certains processus de changement grammatical que les constructions progressives de l'anglais expérimentent tout au long de leur développement diachronique. Plus précisément, les processus de grammaticalisation, à la fois primaires et secondaires, dans lesquels deviennent les formes progressives, ainsi que les phénomènes de changement de construction et de constructionnalisation qui semblent avoir lieu dans l'évolution de ces structures, sont étudiés. Une analyse qualitative standardisée est réalisée, à partir d'un échantillon de l'ensemble du corpus York-Toronto-Helsinki-Penn, au moyen de laquelle l'existence d'une relation entre les processus primaires de grammaticalisation et les changements de construction, ainsi qu'entre les phénomènes de grammaticalisation secondaire et de constructionalisation est prouvée. Enfin, le concept de gradient linguistique,
} 
appliqué à ces processus de changement grammatical, explique provisoirement la manière dont ces phénomènes de changement pourraient être trouvés latents sous des formes progressives dans toutes les périodes de la langue.

MOTS CLÉS : linguistique historique anglaise, grammaticalisation, changement de construction, constructionnalisation, linguistique de corpus, méthodologie qualitative.

Recibido: 09/09/2019. Aceptado: 04/10/2020

\section{Introducción}

Los estudios actuales más comprensivos sobre las construcciones progresivas del inglés explican la evolución de estas estructuras a la luz de diversos procesos de gramaticalización (cf. Núñez-Pertejo 2004; Smitterberg 2005; Smith 2007; Kranich 2010; Killie 2014).

Kranich (2010: 77) sostiene que en inglés contemporáneo o actual (IC) estas construcciones básicamente funcionan como un marcador aspectual que presenta un alto grado de gramaticalización.

En la gramaticalización, la premisa básica es que el proceso está asociado a un incremento en la frecuencia de aparición de las estructuras que la experimentan, ya que la gramaticalización se acompaña de diseminación y expansión contextual, que en inglés se denomina semantic extension.

No obstante, Heine y Kuteva (2005: 45) mantienen que la naturaleza exacta de la interacción entre la frecuencia de uso, la diseminación contextual $\mathrm{y}$ el cambio funcional aún no se ha precisado de forma concluyente. En este sentido, Mair (2006: 138) considera que los aumentos de frecuencia deben relacionarse cualitativamente con los procesos de gramaticalización. En otras palabras, debe detallarse cómo los aumentos de frecuencia son indicativos de gramaticalización. Así, el análisis cualitativo de ocurrencias resulta determinante para hallar evidencias de este tipo de cambio gramatical (cf. Kranich 2010: 17; Petré 2015: 48).

Más recientemente se han desarrollado diversas propuestas sobre el cambio gramatical que comprenden lo que se califica como cambio construccional y construccionalización (Bergs y Diewald 2008; Traugott y Trousdale 2013; Hilpert 2013). Según mi valoración, estos enfoques podrían complementar los modelos 
de gramaticalización con los que tradicionalmente se explica el desarrollo de las estructuras progresivas.

En estos estudios diacrónicos basados en modelos construccionales, el cambio gramatical se describe mediante el análisis de las variaciones que experimentan los pares simbólicos de forma y significado construccional, lo que puede dar lugar a una reorganización parcial del sistema construccional de la lengua, o bien a la aparición de nuevas construcciones lingüísticas para codificar una categoría gramatical concreta.

En estos modelos, las macro-construcciones constituyen el nivel más abstracto de esquematización de los pares simbólicos de forma y significado, y subsumen otros niveles más específicos de esquematización, es decir, las microconstrucciones.

Un ejemplo de macro-construcción es la construcción inglesa de determinación, en la que una estructura nominal concurre con una estructura determinante, ya sea un demostrativo, un pronombre posesivo, un sintagma preposicional con of o un determinante definido. Por su parte, las micro-construcciones especifican formalmente las configuraciones conceptuales de los constructos (cf. Hilpert 2013: 2-8). En el caso de la construcción determinada, un ejemplo de microconstrucción es la construcción de determinación definida, article $N$, que se realiza en el constructo the house.

Las construcciones varían funcional y estructuralmente. Esto produce, tanto en las micro-construcciones como en las macro-construcciones, cambios morfosintácticos, procesos de lexicalización y cambios en sus frecuencias relativas de aparición.

En el caso concreto de las construcciones progresivas, considero que los procesos de cambio construccional y construccionalización tienen lugar a la par de los fenómenos de gramaticalización. De hecho, como se verá en detalle más adelante, determinados desarrollos característicos de la construccionalización se producen en la evolución de las formas progresivas. En una primera aproximación, es notorio el proceso de lexicalización de la macro-construcción para codificar oposiciones semánticas abstractas; es decir, aspecto progresivo frente a no progresivo, así como la redistribución concomitante de las frecuencias relativas de aparición de las estructuras y funciones que codifican esta categoría aspectual (cf. van Arkel-Simón 2018).

En aras de probar esta hipótesis inicial, este estudio pretende ilustrar cualitativamente los procesos de gramaticalización en que devienen las 
estructuras progresivas, así como los fenómenos de cambio construccional y construccionalización que, según mi criterio, paralelamente experimentan estas formas a lo largo de su evolución diacrónica. Para llevar a cabo el estudio, se emplea una muestra del conjunto de corpus York-TorontoHelsinki-Penn en la que se aplican sendas rúbricas de análisis estandarizadas, una para los procesos de gramaticalización, y otra para los fenómenos de cambio construccional y construccionalización. Más concretamente, se estudian diversos prototipos progresivos del inglés antiguo (en adelante IA), el inglés medio (en adelante IM), el inglés moderno temprano (en adelante IMT) y el inglés moderno tardío (en adelante IModT) en virtud de las tendencias de relación significativas que muestran los datos del grupo de corpus, específicamente, con los fenómenos de gramaticalización (cf. van Arkel-Simón 2018).

\section{Fenómenos de cambio gramatical en las construcciones progresivas}

\subsection{Los procesos de gramaticalización y subjetivación}

La gramaticalización es el proceso de cambio lingüístico mediante el cual formas que inicialmente presentan carácter léxico adquieren carácter gramatical y estas últimas, a su vez, adquieren mayor carácter gramatical. Es decir, las estructuras que experimentan este proceso cumplen funciones cada vez más típicamente gramaticales y su significado resulta cada vez más paradigmático (cf. Heine y Kuteva 2002: 2). Los procesos de gramaticalización, según Kranich (2010: 5), se caracterizan por cuatro mecanismos principales:

1) Pérdida de contenido semántico (semantic bleaching).

2) Diseminación o generalización contextual (semantic extension).

3) Pérdida de propiedades morfosintácticas (decategorialization).

4) Erosión fonética (attrition/ erosion).

Asimismo, Lehmann (2002: 146) establece que los procesos que ocurren en la gramaticalización pueden identificarse en los ejes sintagmático y paradigmático. Según este autor, los procesos característicos del eje paradigmático son la pérdida de rasgos semánticos (desemantization), la erosión fonética (erosion), la inclusión en un determinado paradigma (paradigmaticization), y el uso obligatorio en determinados contextos (obligatorification). Los procesos característicos del eje sintagmático son, 
según este mismo autor, la pérdida de características morfosintácticas (condensation), la coalescencia y consolidación estructural (coalescence), y la fijación de la posición sintáctica (fixation).

Como sostiene Kranich (2010: 6-7), las construcciones progresivas han experimentado un largo proceso diacrónico de gramaticalización. Así, el desarrollo de los marcadores de aspecto, tiempo y modo se entiende como un largo proceso de cambio que comprende fenómenos de gramaticalización primaria, en un principio, y fenómenos de gramaticalización secundaria, más adelante. La gramaticalización primaria de las construcciones progresivas comprende el desarrollo de funciones sintácticas, es decir, en la perífrasis verbal el verbo to be adquiere su carácter actual de auxiliar. Según Traugott (1992: 188), este primer proceso podría haber comenzado desde el período del IA.

En la gramaticalización secundaria, que ocurre posteriormente, la construcción adquiere un significado gramatical determinado y establece relaciones paradigmáticas con formas gramaticales alternativas, en otras palabras, pasa a formar parte integral del sistema auxiliar de la lengua. Este proceso de gramaticalización secundaria, según la literatura especializada, tiene lugar en el período moderno de la lengua (cf. Fischer 1992: 254; Denison 1993: 407).

Existe una relación probada entre los procesos de gramaticalización y el fenómeno de la subjetivación (Traugott 1990: 500). Mediante la subjetivación, los significados expresan cada vez más la percepción subjetiva del emisor con respecto a la situación denotada. La gramaticalización primaria, que ocurre en las primeras etapas, se acompaña del fenómeno de la subjetivación: gracias al enriquecimiento pragmático de las construcciones que produce la subjetivación tiene lugar la gramaticalización (cf. Traugott 2003: 71-98; Fischer 2007: 259). Obsérvese, por ejemplo, la subjetivación que muestra en IA este ejemplo del participio wuniende, del verbo léxico wunian ('habitar', 'morar', 'permanecer'):

(1) Seo pridde yld was. pa wuniende oð Dauid

[The] [poisonous] [maturity] [was] [then] [dwelling] [on] [David]

La venenosa vejez estaba prematuramente habitando en David

(colsigewZ,+ALet_4_[SigeweardZ]:270.92) ${ }^{2}$

(2) an anre Godcundnysse afre wuniende, on anum magen

[a] [single] [Godhead] [always] [dwelling] [on] [an] [own]

2. Ocurrencia extraída del género de los Tratados según la propuesta de reclasificación de los géneros textuales del conjunto York-Toronto-Helsinki-Penn que establece van Arkel-Simón (2018). 


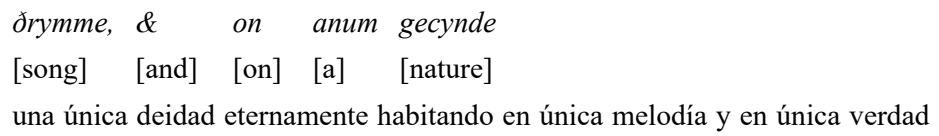

En los estadios iniciales de su proceso de gramaticalización; es decir, durante los períodos medievales, las construcciones progresivas presentan unos usos expresivos basados en la percepción subjetiva del emisor en lugar de funciones puramente gramaticales. En otras palabras, presentan unas funciones expresivas que contrastan con la función típicamente gramatical de marcador aspectual atestiguada en inglés moderno. Durante el proceso de gramaticalización secundaria, las estructuras tienden a mostrar un desarrollo opuesto que Kranich (2010: 8-9) denomina objetivación; es decir, las funciones se basan en menor grado en la perspectiva subjetiva o actitudinal del hablante en relación con la proposición. Esta objetivación puede apreciarse en el ejemplo siguiente también para el participio wuniende del IA, el cual pudo influir en el establecimiento de la forma del IM dwelling, además de la forma $d w e l l e n d e$ ('habitando', 'morando', 'viviendo', 'permaneciendo'):

(3) $Đ a$ on westenum wuniende woruldice estas

[Deer] [on] [desert] [dwelling] [earthly] [grace]

Manso en el desierto habitando la gracia terrena

(cocathom1,+ACHom_I,_36:489.104.7204) ${ }^{4}$

(4) dwelling in pe Hospital in meche welth \& prosperyte

[dwelling] [in] [the] [Hospital] [in] [much] [wealth] [and] [prosperity]

habitando en la Orden con mucha riqueza y prosperidad

$(\mathrm{CMKEMPE}, 95.2160)^{5}$

Cuando la construcción establece su función gramatical y sus relaciones paradigmáticas prototípicas en inglés moderno, se pierden los usos subjetivos, o bien disminuyen los contextos que permiten estos usos subjetivos. Así, una vez que la función típicamente gramatical queda establecida, se pierde la expansión funcional que se constata en los significados subjetivos y actitudinales. En otras palabras, aumenta la rigidez morfosintáctica de la construcción y se pierde la complejidad semántica y pragmática en ciertos usos (cf. Heine y Reh 1984: 67).

3. Ejemplo extraído del género de las Homilías (ibíd.).

4. Ejemplo extraído del género de las Homilías (ibíd.).

5. Ocurrencia extraída del género de las Tratados (ibíd.). 


\subsection{Los procesos cambio construccional y construccionalización}

En la construccionalización la paradigmatización es fundamental, pues el surgimiento de un nuevo paradigma gramatical se refleja en la aparición y consolidación de una nueva macro-construcción, la cual configura un nuevo par simbólico de forma y significado. La emergencia de esta nueva esquematización conceptual en la lengua tiene lugar mediante la asimilación formal y funcional de diversos cambios construccionales que, por su parte, ocurren en las microconstrucciones (cf. Hilpert 2013: 9-13).

Los cambios construccionales se definen por la expansión pragmática, la semantización, la ruptura de determinados pares de forma y función y diversos cambios distribucionales que experimentan las micro-construcciones. Por su parte, la construccionalización se define por la expansión de las colocaciones, la reducción morfológica y fonética y la aparición de nuevas oposiciones semánticas en una macro-construcción (cf. Traugott y Trousdale 2013: 27).

Por ejemplo, a partir de una serie de cambios construccionales en IA e IM hlot ('lot') desarrolla su función partitiva y, posteriormente, cuantificadora. En IA, este nominal denotaba un objeto mediante el cual se ungía a una persona para el desempeño de una determinada función social. En IM, gracias a un proceso de extensión metonímica, el significado léxico de lot se amplió a la denotación tanto de la cosa obtenida por esa unción como al destino que determinaba esa unción. En este proceso, una parte implicaba una cantidad, lo que permitió el uso con el significado de grupo:

(5) Azz wass $i$ piss middelloerd Summ lott off gode sawless

[Always] [was] [in] [this] [MiddleEarth] [some] [lot] [of] [good] [souls]

There was always in this world a group of good souls

[c.1200 Ormulum, 19150 (MED lot $\mathrm{n}_{1}, 2$ ), como se cita en Traugott y Trousdale 2013: 24]

Como puede apreciarse en (5), este uso referencial del nominal ocurre en una construcción de tipo relacional: lot es el núcleo de un sintagma nominal y off gode sawless es su complemento de régimen preposicional, asimismo lot refiere a una unidad parte de un todo. La construcción partitiva puede esquematizarse como sigue:

[[Ni [of Nj]] Û [parti - wholej]

(Traugott y Trousdale 2013: 24) 
Posteriormente, en IMT e IModT, se impone un significado cuantitativo que impide el significado partitivo anterior:

(6) She brought down a lot of white rags. I though they seemed quite heavy for their bulk

(1865 Alger, Paul Prescott's Charge (COHA), apud Traugott y Trousdale

2013: 25)

En (6) se observa cómo el significado de la micro-construcción ha cambiado de partitivo a cuantificador, al mismo tiempo que se ha modificado la estructura de constituyentes. Concretamente, se ha producido el reanálisis sintagmático del núcleo sintáctico y el nominal [núcleo nominali $(l o t)>$ núcleo nominalj (white rags)], así como el reanálisis de la preposición of como miembro de la nueva estructura cuantificadora:

\section{[[N of] $\mathrm{Nj}]]$ Û [large quantity - entityj]}

(Traugott y Trousdale 2013: 25)

Esta sucesión de cambios construccionales, desde el partitivo a lot hasta el cuantificador a lot of, resulta en la construccionalización de la estructura, pues se genera un nuevo par gramatical de estructura y función a partir de una serie de micro-construcciones intermedias. Estas micro-construcciones presentan gradualmente mayor carácter gramatical, ya que se pierde paulatinamente contenido léxico-semántico y se fija la estructura morfosintáctica asociada al significado final de la macro-construcción.

Por tanto, la sucesión de cambios construccionales puede resultar en la construccionalización de la estructura si se genera un nuevo par gramatical de estructura y función a partir de una serie de micro-construcciones intermedias. Estas micro-construcciones presentarán gradualmente mayor carácter gramatical, ya que debe perderse paulatinamente contenido léxico-semántico y fijarse la estructura morfosintáctica asociada al significado final de la macro-construcción.

Con todo, los cambios construccionales no siempre dan lugar a la construccionalización. La creación de una nueva construcción implica el establecimiento de un nuevo par de forma y significado entre una estructura gramatical y una función concreta, el cual debe convencionalizarse en los usos de una determinada lengua. Por tanto, los cambios graduales en el grado de 
esquematicidad, productividad y composicionalidad semántico-gramatical que experimentan diversas micro-construcciones pueden afectar únicamente a determinadas dimensiones internas de las construcciones existentes sin llegar a convencionalizar un nuevo par de forma y significado gramatical en una nueva macro-construcción (cf. Traugott y Trousdale 2013: 1-26).

En el contexto de estos procesos puede predecirse la expansión en las colocaciones de la macro-construcción progresiva, así como la reducción fonética y morfológica de la misma. Al mismo tiempo, se espera encontrar los cambios sintácticos y morfológicos graduales característicos del cambio construccional, pues, a lo largo de su evolución, las formas progresivas devienen en diferentes grados de esquematicidad, composicionalidad y productividad semántico-gramatical para dar lugar a la categoría aspectual del inglés contemporáneo. Más específicamente, se estima hallar sucesivos grados de expansión pragmática, semantización, ruptura de los pares de forma y función micro-construccional, así como diversos cambios en la distribución de las estructuras progresivas.

\section{Metodología del estudio}

\subsection{Material y selección de la muestra}

Los corpus lingüísticos pueden constituir una herramienta también idónea para el análisis cualitativo de las tendencias registradas en las construcciones objeto de estudio. En este sentido, el conjunto York-Toronto-Helsinki-Penn es un grupo de corpus históricos equiparables que abarca desde el IA hasta el IModT. El criterio de inclusión de los textos compilados en este conjunto de corpus se basa en el principio de variación socio-histórica, por lo que se estima que sus muestras son representativas de la lengua escrita de cada período. La periodización es de capital importancia en su compilación, así como la variación dialectal, el género textual, el registro empleado por el autor de cada texto y la variación sociolingüística (cf. Kroch y Taylor 2000; Taylor et al. 2003; Kroch, Santorini y Diertani 2004; Kroch et al. 2010).

En el grupo de corpus York-Toronto-Helsinki-Penn, para cada período de la lengua, los géneros textuales que se indican seguidamente constituyen el punto de intersección gráfica o convergencia entre los aumentos más acusados de los Coeficientes-M y $-\mathrm{V}$ de las formas progresivas; las variantes sintácticas de estas estructuras que resultan significativas en diversos modelos 
predictivos; la ratio clase-tipo léxica progresiva estimada; y los cambios más pronunciados en las dimensiones funcionales significativas del modelo multidimensional (MD) en relación con los procesos de gramaticalización (cf. van Arkel-Simón 2018) ${ }^{6}$ :

1) Homilías para el período del IA.

2) Tratados para el período del IM.

3) Biografía para el período del IMT.

4) Biografía para el período del IModT.

Estos géneros textuales contienen 11.480 ocurrencias progresivas para el análisis. Así, los géneros seleccionados se corresponden con las secciones del conjunto de corpus en las que la confluencia de las variables computables que resultan significativas en los procesos de gramaticalización, según se establece en van Arkel-Simón (2018), presentan los aumentos más acusados. Se dispone así de una metodología de análisis cualitativo estándar, pues se seleccionan los géneros en los que se ha probado estadísticamente la existencia de una relación significativa entre las variables cuantificables que intervienen en los procesos de gramaticalización.

\subsection{Modelo de análisis cualitativo}

Para analizar los patrones de cambio gramatical en las construcciones progresivas del inglés, se emplearon sendas rúbricas de análisis estandarizadas. ${ }^{7}$ En función de estas rúbricas, se llevó a cabo la identificación de los rasgos correspondientes a los procesos de gramaticalización, tanto primaria como secundaria (cf. 2.1.), así como de los rasgos correspondientes a los fenómenos de cambio construccional y construccionalización (cf. 2.2.).

6. El Coeficiente-M se utiliza para contabilizar la forma progresiva como rasgo lingüístico autónomo (cf. Smitterberg 2005: 40). El Coeficiente-V computa el número de formas progresivas con el número de sintagmas verbales sin excluir las formas verbales que no pueden ocurrir en la forma progresiva. (cf. Smitterberg 2005: 44). Por su parte, la ratio clase-tipo léxica resulta de la división del número distintivo de tipos léxicos progresivos estimados en cada uno de los corpus entre el número total de ocurrencias progresivas extraídas de cada corpus. Por otra parte, para analizar cuantitativamente los cambios en la distribución funcional de las estructuras progresivas, puede emplearse el método estadístico multifactorial o multidimensional (MD) (cf. Biber 1988; Biber y Finegan 1997; Geisler 2002). Este método básicamente consiste en la construcción de una serie de dimensiones funcionales a partir de características morfosintácticas presentes en los corpus en función del género textual.

7. Las rúbricas estandarizadas para el análisis cualitativo se muestran en el anexo de este trabajo. 


\section{Interpretación de los resultados}

\subsection{La gramaticalización primaria y el cambio construccional en las formas progresivas}

Un proceso de gramaticalización primaria debe caracterizarse, en primer lugar, por la aparición de las funciones especiales que confieren subjetivación a la proposición en la que aparecen las estructuras progresivas. Esta subjetivación se registra de forma señalada en los períodos medievales del inglés. Por tanto, en el caso del IA y el IM, esto indica la presencia de gramaticalización primaria. En estos períodos, asimismo asumí la existencia de cambio construccional.

Con el objeto de ilustrar en detalle los citados procesos, presento seguidamente dos prototipos progresivos que permiten el análisis del verbo léxico del IA dwellan ('vagar', 'deambular', 'descarriar', 'ir por mal camino') en su transformación en el verbo léxico del IM dwellen ('habitar', 'morar', 'vivir', 'permanecer'). Nótese que extraje el prototipo correspondiente al IA del género de las Homilías y el prototipo correspondiente al IM del género de los Tratados (cf. 3.1.):

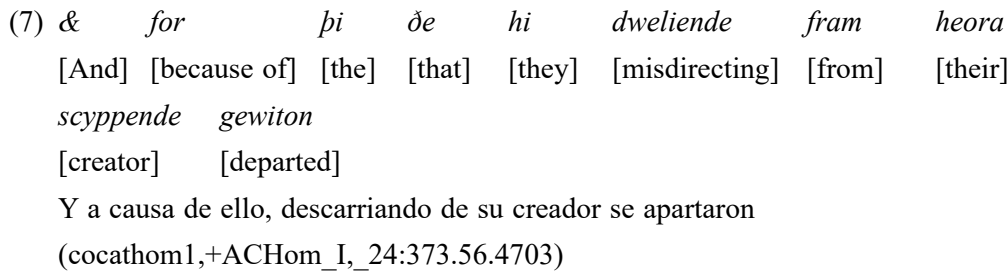
(8) for he had ben dwellyng long tyme wyth an ankyr [because] [he] [had] [been] [dwelling] [long] [time] [with] [an] [anchor] porque él había estado morando mucho tiempo inquebrantable (CMKEMPE,33.727)

En la frase de participio del IA, que cumple una función de complemento circunstancial de modo, dweliende fram heora scyppende gewiton ('descarriando de su creador se apartaron'), se registran usos no aspectuales, más concretamente, una función descriptiva (cf. Núñez-Pertejo 2004: 29-30). Asimismo, puede distinguirse el propósito narrativo no especializado (cf. Smitterberg 2005: 1113). Gramaticalmente, se trata de una frase subordinada de participio que se 
inserta tras la estructura causal \& for pi ðe hi...gewiton ('Y a causa de ello, ....se apartaron').

La forma progresiva verbal conjugada del ejemplo en IM had ben dwellyng ('había estado morando') muestra una mayor subjetivación en el significado de la construcción progresiva. De esta forma, puede identificarse la prominencia de las funciones especiales. Así, a pesar de encontrar las funciones aspectuales prototípicas de las formas verbales; en otras palabras, dinamismo, duración limitada, temporalidad y no completitud; así como ciertas funciones no aspectuales; es decir, habitualidad y expresión de contenido emocional; la subjetividad mediante especificación adverbial es el uso especial que da lugar a la subjetivación de la construcción progresiva en este ejemplo del IM (cf. Núñez-Pertejo 2004: 21-38; Kranich 2010: 44-72). La especificación adverbial característica de este uso especial tiene lugar mediante la locución long tyme ('mucho tiempo'). Asimismo, la presencia de la locución preposicional de tipo interpretativo wyth an ankyr ('inquebrantable') contribuye a la subjetivación de la proposición en la que se inserta la forma progresiva.

En este prototipo del IM, se observa también una función narrativa no especializada, algo que es especialmente relevante en el género de los Tratados, pues este género presenta una tendencia funcional opuesta en el conjunto de corpus York-Toronto-Helsinki-Penn (cf. van Arkel-Simón 2018). Gramaticalmente, la perífrasis progresiva constituye el núcleo del sintagma verbal en una estructura causal: for he had ben dwellyng ('porque él había estado morando').

Un análisis aspectual más detallado del prototipo verbal del IM indica que, con respecto al aspecto gramatical, nos encontramos ante un verbo imperfectivo, dwellen ('habitar', 'morar', 'vivir', 'permanecer'), que aparece en la forma progresiva gracias a la focalización en un alcance inmediato de la predicación: dwellyng ('habitando', 'morando', 'viviendo', 'permaneciendo') (cf. Langacker 1996: 290-293). Esto nos permite concebir una configuración que se repite a lo largo del tiempo. En este sentido, en relación con el aspecto léxico, nos hallamos ante un estado conceptualizado como un estado temporal habitual. Esta configuración se debe a la conceptualización del verbo estativo primario dwellen ('habitar', 'morar', 'vivir', 'permanecer') como una sucesión de situaciones equivalentes (cf. Radden y Dirven 2007: 190-195). Asimismo, este prototipo se corresponde con una construcción progresiva de alteración de tipos explícita, pues se produce la coerción del verbo de carácter estativo para codificar la categoría semántica de actividad que requiere la construcción en el participio presente. El núcleo auxiliar de tipo perfecto had ben ('había estado') codifica un estado que 
se mantiene mientras dura la actividad que expresa el complemento sintagmático verbal dwellyng ('morando'). Estas últimas especificaciones configuran lo que denomino, siguiendo a Michaelis (2005), el aspecto léxico-construccional de esta forma progresiva verbal.

En estos ejemplos del inglés medieval se registran también muchos de los parámetros de gramaticalización establecidos por Lehmann (2002: 121-178). En primer lugar, en el eje paradigmático, se produce la desemantización del significado del verbo léxico primario, pues se restringen las denotaciones que presenta el verbo en su tránsito del IA al IM. Al mismo tiempo, puede apreciarse el proceso de reanálisis morfológico que experimenta la forma de participio presente: $d w e l i e n d e>d w e l l y n g$. Más específicamente, se registra la reducción fonética del afijo: ende> yng. Asimismo, existe una mayor homogeneidad paradigmática en la estructura del IM, pues aumentan las relaciones de complementariedad posibles en este período. Estas relaciones quedan ilustradas por la aparición de la forma perfecta del participio verbal had ben dwellyng. Así, es posible establecer nuevos contrastes semántico-gramaticales que resultan en nuevas relaciones de complementariedad con el resto de formas progresivas conjugadas, las cuales requieren la participación del resto de miembros del grupo auxiliar verbal para su configuración.

Esta descripción es indicativa de que se está empezando a constituir una categoría gramatical obligatoria para codificar unas funciones específicas. De este modo, de acuerdo con las propuestas de Fischer (1992: 233-278), se observa que la constitución del sistema auxiliar del inglés condiciona la obligatoriedad de la forma de participio presente para codificar la función aspectual. Como puede apreciarse, el proceso se caracteriza por la desemantización del participio, pues se pierde parte de su carácter léxico; la erosión fonética de esta forma verbal, la cual tiene lugar por el proceso de reanálisis morfológico antedicho; y el establecimiento de nuevas relaciones contrastivas, expresadas por la complementariedad que muestran las formas conjugadas en el conjunto del grupo auxiliar verbal.

En el eje sintagmático, la capacidad para proyectar constituyentes disminuye en el prototipo de participio presente del IM, pues ha tenido lugar su condensación con el resto del sistema auxiliar verbal. En otras palabras, puede observarse el proceso de reanálisis que ha experimentado el participio presente con el resto de miembros de la categoría auxiliar. En este sentido, las posibilidades de proyección sintáctica del participio presente como categoría verbal independiente han disminuido en IM, observándose un ajuste de posiciones que configura una situación de alternancia sintagmática más restringida. Por su parte, puede 
advertirse también el fenómeno de coalescencia que ha experimentado el participio presente, el cual se manifiesta por su grado de cohesión con el resto del sistema auxiliar verbal. Por último, se constata la reducción fonética del participio, concretamente, mediante la aglutinación de los afijos de las formas de participio presente en -yng. Probablemente, esta reducción fonética es motivada por un proceso de analogía.

Según mi valoración, este ejemplo de gramaticalización primaria constituye asimismo un caso de cambio construccional, pues se registran muchos de los parámetros establecidos por Traugott y Trousdale (2013: 22-26). En este sentido, en los ejemplos del IA e IM puede apreciarse la expansión pragmática que experimenta la micro-construcción progresiva. Esto queda ilustrado por la prominencia de las funciones descriptivas y especiales que implican la subjetivación de las proposiciones en las que aparece. Al mismo tiempo, se produce la semantización de la construcción como unidad especializada de significado, pues gradualmente representa una unidad paradigmática que comienza a ser distintiva y obligatoria en el sistema aspectual de la lengua. Es decir, la construcción comienza a especializarse para codificar obligatoriamente la categoría aspectual progresiva del inglés.

Además, el participio presente ha experimentado un proceso de reanálisis morfológico donde se observa también una reconfiguración sintáctica, la cual se produce en función del resto de elementos del sistema auxiliar verbal. De este modo, como se verá más adelante, la micro-construcción progresiva dará lugar a una macro-construcción especializada en el sistema gramatical de la lengua. Así, los cambios relativos en las frecuencias de aparición de las variantes sintácticas progresivas en estos períodos probablemente se deban a estos procesos de reconfiguración de las relaciones estructura-función, lo que finalmente dará lugar a la especialización de la construcción progresiva como una macro-construcción de carácter aspectual del inglés (cf. van Arkel-Simón 2018).

\subsection{La gramaticalización secundaria y la construccionalización en las formas progresivas}

Un fenómeno de gramaticalización secundaria debe identificarse, en primer término, por la presencia de una mayor rigidez gramatical que debería asociarse también con la aparición de una mayor objetivación de la predicación en la que ocurre la forma progresiva. De este modo, debería registrarse una restricción funcional en la que disminuya la presencia de los fenómenos de 
subjetivación. Esta es la situación concreta que tiene lugar en los períodos del IMT e IModT. En este contexto, el mayor grado de fijación gramatical propio de estos períodos sugiere que, de forma concomitante, están produciéndose procesos de construccionalización. Así, una etapa de gramaticalización primaria y cambios construccionales da lugar a una mayor estabilidad en el sistema aspectual del inglés, lo cual no restringe las posibilidades de cambio gramatical en estos períodos.

En los prototipos progresivos siguientes trataré de ejemplificar los citados fenómenos de gramaticalización secundaria y construccionalización. Los prototipos seleccionados son ocurrencias del verbo léxico go en su evolución del IMT al IModT. Ambos prototipos progresivos han sido extraídos del género de la Biografía (cf. 3.1.):

(9) Sir John Walter is going to be marryed to my Lady Stoel, which will be very happy for him

Sir John Walter se va a casar con Lady Stoel, lo que seguro le hará muy feliz

(ANHATTON-E3-H,2,214.42)

(10) and very great was my pleasure in going over the house and grounds mayúsculo fue mi placer al ir revisando la casa y las tierras

(AUSTEN-180X,168.182)

En la estructura perifrástica de infinitivo del complejo verbal en el ejemplo del IMT is going to be marryed, se observan funciones aspectuales; es decir, dinamismo, duración limitada, temporalidad y no completitud; usos no aspectuales; esto es, expresión de tiempo futuro y función descriptiva; así como usos especiales, concretamente, carácter de sujeto agente, tipo de situación manifiesta y subjetividad sin especificación adverbial (cf. Núñez-Pertejo 2004: 21-35; Kranich 2010: 54-68; van Arkel-Simón 2019). La presencia de la estructura subordinada adjetiva which will be very happy for him contribuye a la aparición de esta subjetividad. Asimismo, se registra un propósito narrativo no especializado y un uso de tipo conversacional (cf. Smitterberg 2005: 12).

En relación con el componente gramatical del aspecto, el tipo verbal primario del IMT constituye un proceso perfectivo que al adoptar la forma progresiva se convierte en imperfectivo (cf. Langacker 1996: 290). Esta configuración tiene lugar mediante la conceptualización metonímica del verbo perfectivo primario en 
la perífrasis progresiva going to be married. La especificación de esta metonimia se corresponde con el tipo resultado por acción (que lleva al resultado). Por su parte, en relación con el componente léxico del aspecto, se trata de un evento que es conceptualizado como una actividad de culminación (cf. Radden y Dirven 2007: 188-189). En esta actividad resulta prominente la duración del evento, así como su carácter puntual. La duración viene codificada por la perífrasis progresiva going to que extiende en el tiempo la puntualidad del logro que representa el verbo perfectivo primario be married. Así, la focalización en un marco de visualización restringido excluye la fase de realización del logro, pues el punto final de la actividad de culminación se encuentra fuera del mismo. Por tanto, la telicidad es codificada principalmente por la semántica del verbo perfectivo primario be married. En este ejemplo verbal prototípico del IMT, la construcción progresiva constituye una muestra de la construcción de alteración de tipos implícita, pues la construcción no ejerce coerción sobre el tipo verbal perifrástico de infinitivo en el que se forma el participio presente going to, al existir concordancia con la categoría semántica actividad que requiere la construcción. El segundo miembro de valencia de ésta, el núcleo auxiliar be, codifica un estado que se mantiene durante la duración de la actividad que especifica el participio presente (cf. Michaelis 2005: 72-76). Estas especificaciones precisas configuran lo que denomino el componente léxico-construccional del aspecto de este prototipo progresivo (cf. 4.1.).

Por su parte, en la estructura preposicional subordinada de participio del verbo frasal del ejemplo en IModT in going over, se registran funciones no aspectuales; es decir, función descriptiva. Asimismo, se observa el propósito narrativo no especializado. Como puede apreciarse, se produce la objetivación de los significados en los períodos del IMT y el IModT, lo que caracteriza el proceso de gramaticalización secundaria que ha tenido lugar. Así, en el eje paradigmático, la desemantización del contenido léxico del participio presente se ha completado en estos períodos, de modo que la macro-construcción progresiva verbal se encuentra en una relación de complementariedad paradigmática plena como construcción aspectual. De hecho, la construcción en IMT constituye una categoría ya completamente obligatoria para codificar la función aspectual progresiva.

En el eje sintagmático el alcance de la estructura de constituyentes que puede proyectar el participio presente es mínimo en los períodos modernos. Asimismo, el proceso de coalescencia morfológica que ha experimentado el participio presente; en síntesis, gangende> goynge> going; así como el proceso 
de reanálisis sintáctico que ha experimentado la construcción, el cual tiene lugar en función del resto de miembros de la categoría auxiliar, han configurado un ajuste de posiciones que permite, en IModT, una estructura frasal de participio con partícula preposicional, así como una estructura perifrástica de infinitivo en IMT.

Según mi criterio, en estos prototipos del inglés moderno el proceso de construccionalización de la construcción progresiva, en la línea de las propuestas de Traugott y Trousdale (2013: 22-26), viene dado por el fenómeno de reanálisis que da lugar a la fijación de las estructuras de participio en la construcción aspectual del IMT y en la estructura subordinada del IModT. Esta fijación se identifica por la mayor rigidez gramatical que presenta la construcción en estos períodos.

Asimismo, la expansión pragmática ha disminuido con respecto a los períodos anteriores constituyéndose plenamente la macro-construcción aspectual progresiva. Este hecho queda reflejado en la disminución de la subjetividad que confieren las formas progresivas, de modo que se constata la objetivación de las mismas conforme se avanza hacia el IMT y el IModT. La macro-construcción aspectual resultante presenta unas relaciones de oposición semántica plenas y de carácter obligatorio con otras construcciones gramaticales, así como con diversas unidades léxicas, de forma que codifica distintivamente la categoría aspectual progresiva del inglés. El ejemplo más básico de esta relación de oposición lo constituye el par progresivo/ no progresivo, el cual ilustra la especialización de la forma progresiva dentro del núcleo del grupo verbal. Este hecho permite la expansión de las posibilidades de colocación de la construcción progresiva con alternativas léxicas y gramaticales. Así, el grado de esquematicidad y productividad resulta máximo en los prototipos progresivos del IMT y el IModT. De esta forma, la progresiva rigidez gramatical que se observa en la evolución de las estructuras progresivas parece indicativa de que los procesos de gramaticalización, cambio construccional y construccionalización han completado un ciclo de acusado cambio lingüístico en el sistema construccional de la lengua.

\section{Discusión y conclusiones}

Los resultados de este estudio parecen corroborar que la gramaticalización de carácter primario, caracterizada principalmente por la subjetivación en los usos de la construcción progresiva, tiene lugar en los períodos medievales de la 
lengua. Como se ha podido apreciar, mediante la subjetivación los significados expresan en mayor grado la percepción subjetiva del emisor con respecto a la situación denotada (cf. 4.1.).

Del mismo modo, parece confirmarse que la gramaticalización de carácter secundario, que se distingue fundamentalmente por la objetivación en las funciones que desempeñan estas estructuras, tiene lugar en los períodos modernos de la lengua. Así, en los prototipos seleccionados se ha descrito cómo las funciones de la construcción se basan en menor grado en la perspectiva subjetiva o actitudinal del hablante en relación con la proposición (cf. 4.2.).

En consonancia con las propuestas de Lehmann (2002: 146), en los análisis de este estudio se ha mostrado que ambos tipos de gramaticalización se acompañan de la paulatina adquisición de una mayor rigidez morfosintáctica que, a grandes rasgos, se define por el establecimiento de nuevas relaciones paradigmáticas de oposición y complementariedad, así como por la aparición de fenómenos de coalescencia y reanálisis sintagmático.

En mi opinión, el fenómeno de gramaticalización primaria que experimentan las formas progresivas se relaciona con los procesos de cambio construccional propuestos por Traugott y Trousdale (2013: 26). Como se ha podido observar, estos fenómenos de cambio construccional se distinguen, fundamentalmente, por la expansión pragmática, la semantización de la construcción y la aparición de cambios distribucionales en las frecuencias de aparición de estas estructuras (cf. 4.1.).

Según valoración, el fenómeno de gramaticalización secundaria se relaciona, por su parte, con los procesos de construccionalización que describen estos mismos autores (Traugott y Trousdale 2013: 22). En los prototipos analizados, la construccionalización se ha caracterizado por la expansión de las colocaciones de la construcción, la reducción fonética y morfológica y la aparición de nuevas oposiciones construccionales con contenido semántico distintivo (cf. 4.2.).

Por tanto, se confirma la hipótesis de que los fenómenos de gramaticalización, cambio construccional y construccionalización como destacados procesos de cambio gramatical que experimentan las formas progresivas se relacionan positivamente en la muestra analizada.

Con todo, considero que tanto los procesos de gramaticalización como los de cambio construccional y construccionalización en las estructuras progresivas se encuentran latentes en la lengua. Así, estos procesos de cambio gramatical podrían 
constituir un caso de gradación lingüistica que permitiría explicar la prominencia de determinados patrones de cambio en cada período. En este contexto, juzgo deseable ampliar la descripción de las relaciones existentes entre este aparente gradiente en los procesos de cambio gramatical y la evolución morfosintáctica y funcional de las estructuras objeto de estudio. De esta forma, sería finalmente posible interpretar la naturaleza exacta de las relaciones que establecen los patrones gramaticales y funcionales con los procesos de consolidación de los usos construccionales, de modo que, eventualmente, se observen sucesivos ciclos de cambio gramatical.

\section{Bibliografía}

BERGS, A. T. y DIEWALD, G. (2008). Constructions and Language Change. Berlin; New York: Mouton de Gruyter.

DENISON, D. (1993). English Historical syntax: Verbal Constructions. New York: Longman.

FISCHER, O. (1992). "Syntax" en The Cambridge History of the English Language (Vol. II) (Ed. N. Blake). Cambridge: Cambridge University Press: 207-408.

FISCHER, O. (2007). Morphosyntactic Change. Functional and Formal Perspectives. Oxford: Oxford University Press.

HEINE, B. y KUTEVA, T. (2002). World Lexicon of Grammaticalization. Cambridge: Cambridge University Press.

HEINE, B. y KUTEVA, T. (2005). Language Contact and Grammatical Change. Cambridge: Cambridge University Press.

HEINE, B. y REH, M. (1984). Grammaticalization and Reanalysis in African Languages. Hamburg: Helmut Buske.

HILPERT, M. (2013). Constructional Change in English. Cambridge: Cambridge University Press.

KILLIE, K. (2014). "The Development of the English BE + V-ende/ V-ing Periphrasis: from Emphatic to Progressive Marker?". English Language and Linguistics 18 (3): 361-386.

KRANICH, S. (2010). The Progressive in Modern English: A Corpus-Based Study of Grammaticalization and Related Changes. Amsterdam; New York: Rodolpi.

KROCH, A. y TAYLOR, A. (2000). Penn-Helsinki Parsed Corpus of Middle English (PPCME2) (2nd edition). Departamento de Lingüística, Universidad de Pensilvania. CD-ROM, tercer lanzamiento. 
KROCH, A., et al. (2004). Penn-Helsinki Parsed Corpus of Early Modern English (PPCEME) (1st edition). Departamento de Lingüística, Universidad de Pensilvania. CD-ROM, segundo lanzamiento.

KROCH, A., et al. (2010). The Penn Parsed Corpus of Modern British English (PPCMBE) (1st edition). Departamento de Lingüística, Universidad de Pennsylvania. CD-ROM, primer lanzamiento.

LANGACKER, R. W. (1996). "A Constraint on Progressive Generics" en Conceptual Structure, Discourse, and Language (Ed. A. E. Goldberg). San Diego, University of California: Center for the Study of Language and Information: 289-302.

LEHMANN, C. (2002). Thoughts on Grammaticalization. Segunda edición revisada. Erfurt: Seminar für Sprachwissenschaft der Üniversität.

MAIR, C. (2006). Twentieth-Century English: History, Variation, and Standarization. Cambridge: Cambridge University Press.

MICHAELIS, L. A. (2005). "Entity and Event Coercion" en Construction Grammars: Cognitive Grounding and Theoretical Extensions (Eds. J-O. Östman, y M. Fried). Amsterdam; Philadelphia: John Benjamins: 44-88.

NÚÑEZ-PERTEJO, P. (2004). The Progressive in the History of English with Special Reference to the Early Modern English Period: A Corpus-Based Study. München: LINCOM.

PETRÉ, P. (2015). "Grammaticalization by Changing Co-text Frequencies, or Why [BE Ving] Became the Progressive". English Language and Linguistics 20 (1): 31-54.

RADDEN, G. y DIRVEN, R. (2007). Cognitive English Grammar. Amsterdam; Philadelphia: John Benjamins.

SMITH, A. (2007). "The Development of the English Progressive". Journal of Germanic Linguistics 19 (3): 205-241.

SMITTERBERG, E. (2005). The Progressive in 19th-Century English: A Process of Integration. Amsterdam; New York: Rodolpi.

TAYLOR, A., et al. (2003). The York-Toronto-Helsinki Parsed Corpus of Old English Prose (YCOE) (1st edition). Departamento de Lingüística, Universidad de York: Oxford Text Archive. <http://www-users.york. ac.uk/ lang22/YcoeHome1.htm>. (Acceso 11 Junio 2019).

TRAUGOTT, E. C. (1990). "From Less to More Situated in Language: The Unidirectionality of Semantic Change" en Papers from the 5th International Conference on English Historical Linguistics (Eds. S. Adamson, V. Law, N. Vincent y S. Wright). Amsterdam: John Benjamins: 496-517.

TRAUGOTT, E. C. (1992). "Syntax" en The Cambridge History of the English 
Language (Vol. II) (Ed. R. Hogg). Cambridge: Cambridge University Press: 168-289.

TRAUGOTT, E. C. (2003). "Constructions in Grammaticalization" en The Handbook of Historical Linguistics (Eds. B. Joseph y R. Janda). Oxford: Blackwell: 624-647.

TRAUGOTT, E. C. y TROUSDALE, G. (2013). Constructionalization and Constructional Changes. Oxford: Oxford University Press.

VAN ARKEL-SIMÓN, C. (2018). "Modelos estadísticos para el análisis funcional de los procesos de gramaticalización en las construcciones progresivas del inglés". EPOS 34: 333-355.

VAN ARKEL-SIMÓN, C. (2019). “Análisis cualitativo-funcional comparativo para el estudio de los procesos de gramaticalización en las construcciones progresivas del inglés". Revista de Filología 39: 77-94. 


\section{ANEXO: RÚBRICAS ESTÁNDAR PARA EL ANÁLISIS CUALITATIVO DE LOS FENÓMENOS DE CAMBIO GRAMATICAL EN LAS CONSTRUCCIONES PROGRESIVAS DEL INGLÉS.}

1. Rúbrica estándar para el análisis de la gramaticalización primaria y secundaria.

1. Main category (verbal progressive, adjectival progressive or nominal progressive):
1. M-Coefficient:
2. lexical type-token ratio:
3. textual genre:

ADDITIONAL REMARKS:

2. Subjectification (pragmatic enrichment: primary grammaticalization)/ Objectification (pragmatic loss: secondary grammaticalization):
a. Semantic-pragmatic extension (expansion) (High/ Low):
1. non-aspectual functions $(\mathrm{Y} / \mathrm{N})$
2. special functions $(\mathrm{Y} / \mathrm{N})$
3. grammatical function $(\mathrm{N} / \mathrm{Y})$

ADDITIONAL REMARKS:

3. Obligatorification (systemic relevance: aspectual function)/ Noobligatorification (optional function):
a. trans-paradigmatic alternance (grammatical category) (Low/ High)
b. intra-paradigmatic alternance (alternative oppositions) (Low/ High) ADDITIONAL REMARKS:

4. Paradigmaticization/ No-paradigmaticization:
a. complementary distribution (paradigmatic homogeneity) (High/ Low)
b. contrastive distribution (arbitrariness) (High/ Low)

ADDITIONAL REMARKS: 
5. Desemantization (integrity loss) / No-desemantization:

a. semantic bleaching or depletion (High/ Low)

b. phonological attrition (phonetic erosion) $(\mathrm{Y} / \mathrm{N})$

ADDITIONAL REMARKS:

6. Coalescence (bondedness gain)/ No-coalescence:

a. cohesion (juxtaposition) (High/ Low)

b. agglutination (fusion) (High/ Low)

ADDITIONAL REMARKS:

7. Fixation/ No-fixation:

a. syntagmatic position (alternance) (Low/ High)

b. syntagmatic variability (positional adjustment) (Low/ High)

ADDITIONAL REMARKS:

8. Condensation/ No-condensation:

a. constituent structure (structural scope) (Low/ High)

b. explicitness (rematicity) (Low/ High)

ADDITIONAL REMARKS:

9. Reference to relevant contextualized corpus example:

ADDITIONAL REMARKS:

10. Reference to books/ articles, papers, reports, etc. that have studied this progressive form:

ADDITIONAL REMARKS:

11. Model first completed by:

Date:

Revised by*:

Date:

*(enter a new line for each revision)

ADDITIONAL REMARKS: 
2. Rúbrica estándar para el análisis del cambio construccional y la construccionalización.

1. Main category (verbal progressive, adjectival progressive or nominal progressive):

1) Gradualness of constructional development (grammatical change) (Y/N)

2) Conventionalized constructional meaning (Constructionalization) ( $Y / N)$ ADDITIONAL REMARKS:

2. Semantic-pragmatic internal dimensions (Micro-construction):

1) Pragmatic expansion $(Y / N)$

2) Micro-construction semanticization (High/ Low)

ADDITIONAL REMARKS:

3. Morphosyntactic-functional internal dimensions (Micro-construction):

1) Mismatch between micro-constructional form and meaning $(\mathrm{Y} / \mathrm{N})$

2) Functional and structural distributional changes (High/ Low) ADDITIONAL REMARKS:

4. Syntactic change and derivational morphology (Micro-construction reanalysis):

1) Micro-constructional schematicity (High/ Low)

2) Micro-constructional productivity (High/ Low)

3) Micro-constructional compositionality (Low/ High)

ADDITIONAL REMARKS:

5. Lexicalization (Macro-construction):

1) Expansion of macro-constructional collocations (High/ Low)

2) Macro-constructional semantic oppositions (High/ Low) ADDITIONAL REMARKS: 
6. Morphosyntactic-functional and phonetic changes (Macro-construction reanalysis):

1) Morphological and phonological reduction $(\mathrm{Y} / \mathrm{N})$

2) Macro-constructional paradigmaticization (High/ Low)

3) Functional and structural distributional changes (Low/ High)

4) Periphrastic macro-constructional form $(\mathrm{Y} / \mathrm{N})$

ADDITIONAL REMARKS:

7. Reference to relevant contextualized corpus example: ADDITIONAL REMARKS:

8. Reference to books/ articles, papers, reports, etc. that have studied this progressive form:

ADDITIONAL REMARKS:

9. Model first completed by:

Date:

Revised by*:

Date:

*(enter a new line for each revision)

ADDITIONAL REMARKS: 